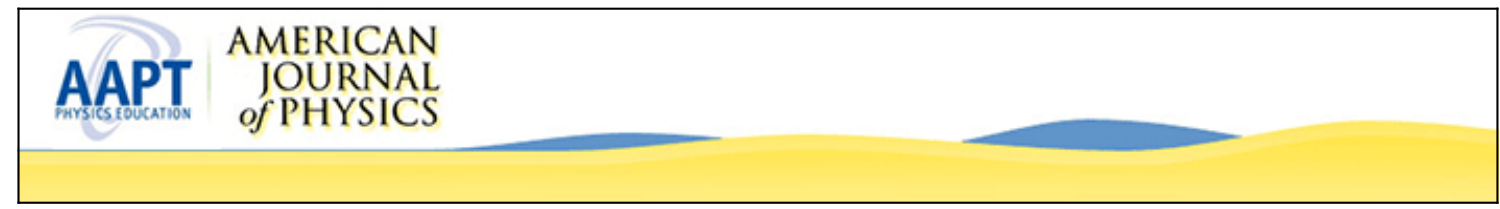

\title{
Thermodynamics of the Adiabatic Expansion of a Mixture of Two Phases
}

M. Cowperthwaite and T. J. Ahrens

Citation: American Journal of Physics 35, 951 (1967); doi: 10.1119/1.1973649

View online: http://dx.doi.org/10.1119/1.1973649

View Table of Contents: http://scitation.aip.org/content/aapt/journal/ajp/35/10?ver=pdfcov

Published by the American Association of Physics Teachers

\section{Articles you may be interested in}

Thermodynamics of adiabatic cross polarization

J. Chem. Phys. 128, 114504 (2008); 10.1063/1.2839436

Two size regimes of methanol clusters produced by adiabatic expansion

J. Chem. Phys. 125, 184303 (2006); 10.1063/1.2378708

Shock Compression, Adiabatic Expansion and Multiphase Equation of State of Carbon AIP Conf. Proc. 620, 759 (2002); 10.1063/1.1483648

Thermodynamic stability of phases and transition kinetics under adiabatic conditions

J. Chem. Phys. 96, 605 (1992); 10.1063/1.462883

Thermodynamic Formulas for TwoPhase Systems

J. Chem. Phys. 26, 909 (1957); 10.1063/1.1743433

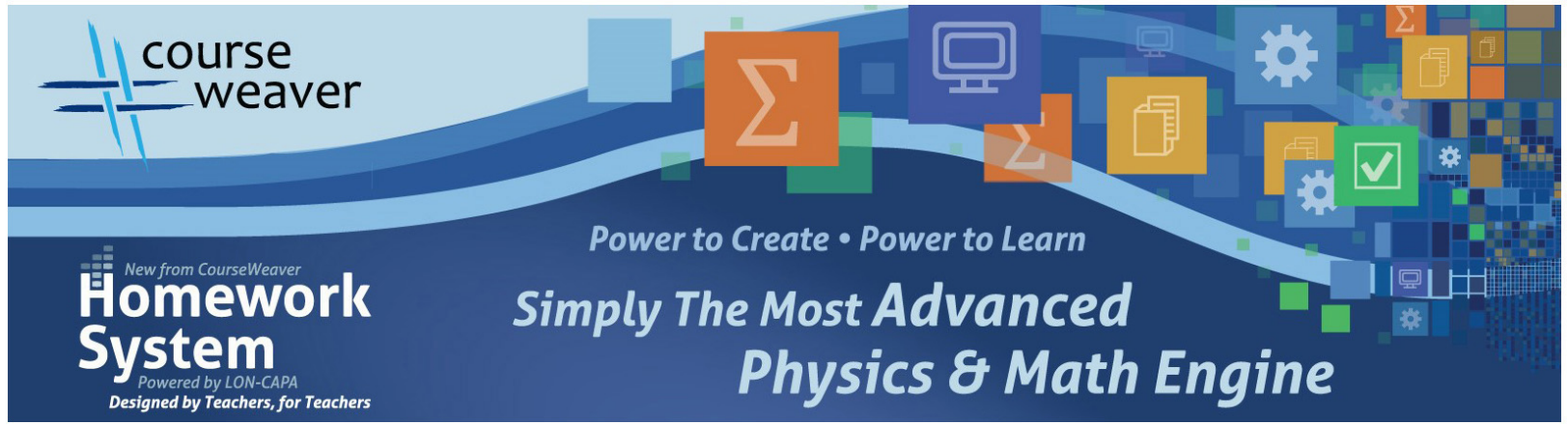




\title{
Thermodynamics of the Adiabatic Expansion of a Mixture of Two Phases*
}

\author{
M. Cowperthwaite and T. J. Ahrens $\dagger$ \\ Stanford Research Institute, Menlo Park, California 94025
}

(Received 27 Apri] 1967)

\begin{abstract}
The thermodynamics of the adiabatic expansion of a mixture of two phases capable of interchanging heat and matter across the phase boundary is presented. The law of conservation of energy is applied to each phase considered as an open system and to the mixture of phases considered as a closed system. Expressions for the entropy production resulting from internal irreversible processes demonstrate the difference between adiabatic and isentropic changes and specify conditions under which the expansion of a closed twophase system is isentropic. Three such possible isentropic processes are defined, and expressions are derived for the temperature-pressure-volume states achieved in them. The thermodynamic treatment is useful in studies of the adiabatic release of a shock-induced mixture of phases.
\end{abstract}

\section{INTRODUCTION}

A THERMODYNAMIC treatment of the adiabatic expansion of a mixture of two phases that are capable of interchanging both heat and matter across the phase boundary is of general interest because it demonstrates the basic principles of irreversible thermodynamics. Expressions for the conservation of energy for the mixture considered as a closed system, and for each phase considered as an open system, ${ }^{1}$ demonstrate different forms of the first law of thermodynamics. Calculation of the entropy production resulting from internal irreversible processes, induced by the expansion and specification of the conditions under which the expansion process is isentropic, demonstrate the second law of thermodynamics.

A knowledge of these isentropic conditions is of special interest in a study of shock-induced phase transitions. If the adiabatic expansion of a shock-induced mixture of phases from the kilobar regime is isentropic, the Reimann invariant ${ }^{2}$ can be used to calculate release adiabats in the pressure-specific volume plane from experimentally determined release adiabats in the pressure-

\footnotetext{
* This research was supported in part by the National Aeronautics and Space Administration under contract NASr-49 (24).

$\dagger$ Present address: California Institute of Technology, Pasadena, Calif. 91105

${ }^{1}$ I. Prigogine, Thermodynamics of Irreversible Processes (Interscience Publishers, Inc., New York, 1961).

${ }^{2}$ R. Courant and K. O. Friedrichs, Supersonic Flow and Shock Waves (Interscience Publishers, Inc., New York, 1948), p. 87.
}

particle velocity plane. Experimental evidence indicates that shock-induced phase transitions occur in quartz, ${ }^{3}$, graphite, ${ }^{4}$ and elemental lead. ${ }^{5}$ Anomalies in the observed shock-induced pressure-volume states of these materials are attributed to the formation of a quartz-stishovite mixture between 144 and $450 \mathrm{kbar}$, of a graphitediamond mixture between 180 and $400 \mathrm{kbar}$, and of a mixture of solid and liquid lead above $245 \mathrm{kbar}$.

\section{THE FIRST LAW OF THERMODYNAMICS FOR THE EXPANSION OF A TWO-PHASE MIXTURE}

For simplicity we consider a system of two phases ( 1 and 2 ) to be in mechanical equilibrium, and assume that external work is performed reversibly by pressure forces. Let the subscripts 1 and 2 denote the state variables of phase 1 and phase 2 , respectively, and let $p$ denote the external pressure. Then the conditions for mechanical equilibrium and for reversible external work are expressed by the equations $p_{1}$ $=p_{2}=p$. The specific internal energy $e$, the specific entropy $s$, and the specific volume $v$ of the mixture are expressed in terms of the specific internal energies, the specific entropies, and the

${ }^{3}$ P. G. McQueen, J. N. Fritz, and S. P. Marsh, J. Geophys. Res. 68, 2319 (1963).

4 B. J. Alder and R. H. Christian, Phys. Rev. Letters 7, 367 (1961).

5 M. H. Rice, R. G. McQueen, and J. M. Walsh, Solid State Phys. 6, 1 (1958). 
specific volumes of the two phases by the equations:

$$
\begin{aligned}
& e=\lambda e_{1}+(1-\lambda) e_{2}, \\
& s=\lambda s_{1}+(1-\lambda) s_{2}, \\
& v=\lambda v_{1}+(1-\lambda) v_{2},
\end{aligned}
$$

where $\lambda$ and $(1-\lambda)$ denote the mass fractions of phase 1 and phase 2 in the mixture.

The two-phase mixture is a closed system but each phase is an open system because it can exchange matter with the other. Application of the first law of thermodynamics to phase 1, phase 2, and the mixture of phases gives

$$
\begin{aligned}
d \lambda e_{1} & =d_{1} \Phi-p d \lambda v_{1}, \\
d(1-\lambda) e_{2} & =d_{2} \Phi-p d(1-\lambda) v_{2}, \\
d e & =d Q-p d v,
\end{aligned}
$$

where $d_{1} \Phi$ denotes the resultant energy that phase 1 receives by heat flow and exchange of matter, $d_{2} \Phi$ denotes the resultant energy that phase 2 receives by heat flow and exchange of matter across the phase boundary, and $d Q$ denotes the heat transferred into the two-phase mixture from the exterior. Elimination of the differentials of the state variables from Eqs. (4), (5), and (6) with the differential forms of Eqs. (1) and (3) gives

$$
d Q=d_{1} \Phi+d_{2} \Phi,
$$

as an expression of the conservation of energy for the two-phase system. Expansion of Eqs. (4) and (5) gives the equations

$$
\begin{gathered}
d_{1} \Phi=\lambda\left(d e_{1}+p d v_{1}\right)+\left(e_{1}+p v_{1}\right) d \lambda, \\
d_{2} \Phi=(1-\lambda)\left(d e_{2}+p d v_{2}\right)-\left(e_{2}+p v_{2}\right) d \lambda,
\end{gathered}
$$

which can be rewritten as

$$
\begin{gathered}
d_{1} \Phi=\lambda d_{1} Q+h_{1} d \lambda, \\
d_{2} \Phi=(1-\lambda) d_{2} Q-h_{2} d \lambda,
\end{gathered}
$$

where $\lambda d_{1} Q$ and $(1-\lambda) d_{2} Q$ denote the heat received by phase $I$ and phase 2 , and $h_{1}$ and $h_{2}$ denote the specific enthalpies of phase 1 and phase 2. It is important to note that the replacement of the $(d e+p d v)$ term in parentheses by the $d Q$ term is valid because the former term contains only intensive variables. Combining
Eqs. (7), (10), and (11) gives a more convenient expression,

$$
d Q=\lambda d_{1} Q+(1-\lambda) d_{2} Q+\left(h_{1}-h_{2}\right) d \lambda,
$$

of the conservation of energy for the two-phase system. Setting $d Q=0$ in Eq. (12) gives the energy equation

$$
\lambda d_{1} Q+(1-\lambda) d_{2} Q+\left(h_{1}-h_{2}\right) d \lambda=0
$$

for an adiabatic change. In this case the energy received by phase 1 from phase 2 is equal to the energy received by phase 2 from phase 1 . Since, in general, $h_{1} \neq h_{2}$, setting $\lambda d_{1} Q=$ $(1-\lambda) d_{2} Q=0$ in Eq. (13) gives $d \lambda=0$ as a characteristic condition for an adiabatic change with no internal heat flow. When there is no internal heat transfer in a mixture of phases during an adiabatic change, the composition of the mixture remains unchanged. Setting $d \lambda=0$ in Eq. (13) gives $\lambda d_{1} Q=-(1-\lambda) d_{2} Q$ as a characteristic condition for a constant composition adiabatic change. When there is no change of composition the heat received by phase 1 from phase 2 balances the heat received by phase 2 from phase 1.

\section{CONDITIONS FOR THE ISENTROPIC EXPANSION OF A TWO-PHASE MIXTURE}

The expression for the entropy production derived from Eq. (13) is sufficient to determine the conditions under which the adiabatic release of a two-phase mixture is isentropic. If $T$ denotes temperature, then the equation for a simple heat transfer $d Q=T d s$ applied to phase 1 and phase 2 gives $d_{1} Q=T_{1} d_{s_{1}}$ and $d_{2} Q=T_{2} d s_{2}$. Substitution of these expressions into Eq. (13) gives

$$
\lambda T_{1} d s_{1}+(1-\lambda) T_{2} d s_{2}+\left(h_{1}-h_{2}\right) d \lambda=0 .
$$

Elimination of the $d s_{2}$ term from Eq. (14) with the equation obtained by differentiating Eq. (2),

$$
d s=\lambda d s_{1}+(1-\lambda) d s_{2}+\left(s_{1}-s_{2}\right) d \lambda,
$$

gives

$T_{2} d s=\lambda\left(T_{2}-T_{1}\right) d s_{1}$

$$
+\left[\mu_{2}-\mu_{1}+\left(T_{2}-T_{1}\right) s_{1}\right] d \lambda,
$$


where $\mu=h-T s$ denotes the specific Gibbs free energy. Equation (16) expresses the entropy production in terms of the internal irreversible processes induced in a mixture of two phases by the adiabatic expansion process. For a mixture of phases that is neither in thermal equilibrium, $T_{1} \neq T_{2}$, nor in chemical equilibrium, ${ }^{6} \mu_{1} \neq \mu_{2}$, the first term in Eq. (16) represents the entropy produced by irreversible heat flow, and the second term represents the entropy produced by an irreversible change of composition. Entropy is conserved either when these two internal processes do not take place or when they take place reversibly. When the adiabatic expansion process is isentropic and $d s=0$ both terms on the righthand side of Eq. (16) must be zero. Three such possible processes are defined below.

\section{A. No Heat Transfer between Phases}

It follows from the first law of thermodynamics, Eq. (13), that internal irreversible processes can occur in the adiabatic expansion of a two-phase mixture only if there is transport of heat between the phases. Thus the adiabatic release process with no internal heat flow is necessarily isentropic. Substituting the conditions for no heat transfer, $d s_{1}=d s_{2}=0$, into Eq. (14) gives $d \lambda=0$, and it follows from Eq. (15) and also from Eq. (16) that $d s=0$.

The position of this type of isentrope in the $(p-v)$ plane is determined by the isentropic conditions for both phases,

$$
\begin{aligned}
& d h_{1}=v_{1} d p=\left(\partial h_{1} / \partial p\right)_{v_{1}} d p+\left(\partial h_{1} / \partial v_{1}\right)_{p} d v_{1}, \\
& d h_{2}=v_{2} d p=\left(\partial h_{2} / \partial p\right)_{v_{2}} d p+\left(\partial h_{2} / \partial v_{2}\right)_{p} d v_{2}
\end{aligned}
$$

and the equation obtained by differentiating Eq. (3) with $\lambda$ constant,

$$
d v=\lambda d v_{1}+(1-\lambda) d v_{2} .
$$

Eliminating $d v_{1}$ and $d v_{2}$ from Eqs. (17), (18), and (19) gives the differential equation

$$
\begin{aligned}
& d v / d p=\lambda\left(\partial v_{1} / \partial h_{1}\right)_{p}\left[v_{1}-\left(\partial h_{1} / \partial p\right)_{v_{1}}\right] \\
& +(1-\lambda)\left(\partial v_{2} / \partial h_{2}\right)_{p}\left[v_{2}-\left(\partial h_{2} / \partial p\right)_{v_{2}}\right]
\end{aligned}
$$

${ }^{6}$ J. W. Gibbs, The Scientific Papers of J. Willard Gibbs: Vol. 1, Thermodynamics (Dover Publications, Inc., New York, 1960). which can be used to construct isentropes if the composition of the mixture and the $(e-p-v)$ equations of state of both phases are known.

Combining the isentropic conditions $d s_{1}=$ $d s_{2}=0$ with thermodynamic identities gives a differential equation for calculating the temperatures of each phase along the isentrope. Let $C_{p}$ and $a$ denote specific heat and volume expansion coefficient at constant pressure. Then, combination of the thermodynamic identities

$$
\begin{gathered}
T(\partial s / \partial T)_{p}=C_{p}=(\partial h / \partial T)_{p}, \\
-(\partial s / \partial p)_{T}=(\partial v / \partial T)_{p}=v \alpha \\
(\partial v / \partial h)_{p}=(\partial v / \partial T)_{p} / C_{p}=\alpha v / C_{p}
\end{gathered}
$$

with the isentropic condition

$$
(\partial s / \partial T)_{p} d T=-(\partial s / \partial p)_{T} d p
$$

gives the differential equation,

$$
d T / T=(\partial v / \partial h)_{p} d p=\left(\alpha v / C_{p}\right) d p,
$$

that governs the variation of temperature with pressure along the isentrope of a single phase. Applying Eq. (22) to each phase gives the differential equations,

$$
\begin{aligned}
& d T_{1} / T_{1}=\left(\alpha v / C_{p}\right)_{1} d p ; \\
& d T_{2} / T_{2}=\left(\alpha v / C_{p}\right)_{2} d p,
\end{aligned}
$$

for calculating the temperatures of each phase during isentropic expansion. Since in general $\left(\partial v_{1} / \partial h_{1}\right)_{p} \neq\left(\partial v_{2} / \partial h_{2}\right)_{p}$ the temperatures of phase 1 and phase 2 will change at different rates along the isentrope. However, as long as the characteristic time for internal heat flow is large compared to the characteristic time for decrease of pressure, the conditions $d \lambda=0, d s_{1}=d s_{2}$ $=0$ will be satisfied, and the adiabatic expansion process will be isentropic.

\section{B. Reversible Heat Transfer between Phases}

The adiabatic expansion of two phases with internal heat flow can only be isentropic if, during the process, the phases are in thermal equilibrium and transfer of heat occurs reversibly. Substituting the condition for thermal equilibrium $T_{1}=T_{2}=T$ into Eq. (16) gives the equation

$$
T d s=\left(\mu_{2}-\mu_{1}\right) d \lambda
$$


for the entropy production for the adiabatic release process with reversible heat flow between phases. The release is isentropic, either when no change of composition takes place, $d \lambda=0$, or when the phases are in chemical equilibrium, $\mu_{2}=\mu_{1}$, and the change of composition takes place reversibly. When the two phases are in thermal equilibrium, formulating the condition that the energy is a perfect differential also gives the equation for the entropy production. In terms of enthalpy, combination of the energy equation for adiabatic changes, $d h=v d p$, with the identity obtained by expanding the $h(s, p, \lambda)$ equation of state,

$$
d h=T d s+v d p+\left(\mu_{1}-\mu_{2}\right) d \lambda,
$$

gives Eq. (25).

\section{No Change of Composition}

Setting $d \lambda=0$ in Eq. (25) gives the isentropic condition $d s=0$ and it follows from Eq. (15) that

$$
\lambda d s_{1}+(1-\lambda) d s_{2}=0 .
$$

The reversible transfer of heat inside the system changes the entropy of each phase in such a way that the total entropy of the system remains constant. The position of an isentrope in the $(p-v)$ plane is determined by the differential equation, $d v=(\partial v / \partial p)_{s, \lambda} d p$

$$
=(\partial v / \partial h)_{p, \lambda}\left[v-(\partial h / \partial p)_{v, \lambda}\right] d p,
$$

obtained by rearranging the isentropic condition,

$$
d h=v d p=(\partial h / \partial p)_{v, \lambda} d p+(\partial h / \partial v)_{p, \lambda} d v \text {. }
$$

The variation of temperature along the isentrope is governed by an equation of the same form as Eq. (22) for a single phase,

where

$$
d T / T=(\partial v / \partial h)_{p, \lambda} d p,
$$

$$
\begin{aligned}
(\partial v / \partial h)_{p, \lambda}=\left[\lambda \alpha_{1} v_{1}+\right. & \left.(1-\lambda) \alpha_{2} v_{2}\right] \cdot \\
& {\left[\lambda C_{p_{1}}+(1-\lambda) C_{p_{2}}\right]^{-1} . }
\end{aligned}
$$

\section{Phases in Chemical Equilibrium}

With the condition for chemical equilibrium $\mu_{1}=\mu_{2}$, Eq. (25) gives $d s=0$ and Eq. (15) reduces to the equation

$$
\lambda d s_{1}+(1-\lambda) d s_{2}+\left(s_{1}-s_{2}\right) d \lambda=0,
$$

which is equivalent to Eq. (13). The entropy flow inside the system, resulting from the reversible transfer of heat and matter, is such that the total entropy of the system remains constant.

An equilibrium isentrope through $\left(p_{i}, v_{i}\right)$ in the mixed phase region of the $p-v$ plane can be constructed with Eqs. (2) and (3) if the positions of the phase boundaries, $v_{1}=v_{1}(p)$, $v_{2}=v_{2}(p)$, and the corresponding entropies along them, $s_{1}=s_{1}(p), s_{2}=s_{2}(p)$, are known. The entropy $s\left(p_{i}, v_{i}\right)$ at $\left(p_{i}, v_{i}\right)$ can be calculated with Eqs. (2) and (3); the $(p-v)$ states on the isentrope through $\left(p_{i}, v_{i}\right)$ must satisfy the equations

$$
\text { and } \begin{gathered}
s\left(p_{i}, v_{i}\right)=\lambda s_{1}(p)+(1-\lambda) s_{2}(p), \\
v=\lambda v_{1}(p)+(1-\lambda) v_{2}(p) .
\end{gathered}
$$

Thus, eliminating the reaction coordinate $\lambda$ between Eqs. $\left(2^{\prime}\right)$ and $\left(3^{\prime}\right)$ gives

$$
\begin{aligned}
& {\left[s\left(p_{i}, v_{i}\right)-s_{1}(p)\right] /\left[v-v_{1}(p)\right]} \\
& \quad=\left[s_{2}(p)-s_{1}(p)\right] /\left[v_{2}(p)-v_{1}(p)\right], \\
& \text { and }
\end{aligned}
$$

$$
\begin{aligned}
& {\left[s\left(p_{i}, v_{i}\right)-s_{2}(p)\right] /\left[v-v_{2}(p)\right]} \\
& \quad=\left[s_{2}(p)-s_{1}(p)\right] /\left[v_{2}(p)-v_{1}(p)\right]
\end{aligned}
$$

as equivalent expressions, in terms of parameters along the phase boundaries, for calculating volume as a function of pressure along an equilibrium isentrope through $\left(p_{i}, v_{i}\right)$. Combining the Clausius-Clapeyron equation,

$$
d p / d T=\left[s_{2}(p)-s_{1}(p)\right] /\left[v_{2}(p)-v_{1}(p)\right],
$$

with Eqs. (30) and (31) gives expressions for the equilibrium isentrope in terms of $(a)$ the entropy and volume along one of the phase boundaries in the $(p-v)$ plane, and $(b)$ the slope of the phase line in the $(p-T)$ plane. It follows that knowledge of the slope of the phase line in the $(p-T)$ plane and of the entropy along one of the phase boundaries in the $(p-v)$ plane is sufficient for constructing the equilibrium isentrope through a point $\left(p_{i}, v_{i}\right)$ of known entropy $s\left(p_{i}, v_{i}\right)$, in the mixed phase region of the $(p-v)$ plane. The temperature along this isentrope can be calculated by integrating Eq. (32), if the slope of the phase line in the $(p-T)$ plane and 
also the temperature at any one point in the mixed region of the $(p-v)$ plane are known.

\section{SUMMARY AND CONCLUSIONS}

This thermodynamic treatment of the adiabatic expansion of a mixture of two phases is based on the assumption that only internal irreversible processes give rise to an increase of entropy. The release process is isentropic, either if internal processes do not occur, or if they occur reversibly. The first and second laws of thermodynamics have been formulated for a mixture of two phases capable of interchanging heat and matter, to determine specific conditions under which the adiabatic expansion process is isentropic. In the case of no heat flow between phases, the adiabatic expansion process is necessarily isentropic because a flow of heat is necessary for a transport of matter and a change of composition. In the case of reversible heat flow between phases the adiabatic expansion process is isentropic either if the composition remains fixed or if the composition changes with the phases in chemical equilibrium. These conditions specify equations for constructing three possible isentropes through a point representing a mixture of phases in the $(p-v)$ plane. The thermodynamic treatment ignores the kinetics of the various processes and therefore cannot be used to determine either if or how an adiabatic change will be isentropic.

However, construction of the various isentropes in the $(p-v)$ plane, and their transformation to the pressure-particle velocity $(p-u)$ plane with the Riemann invariant, are important in shock wave studies. Comparison of calculated and measured release adiabats in the $(p-u)$ plane indicates whether the adiabatic release of a shock-induced mixture of phases is isentropic.

\section{ACKNOWLEDGMENT}

The authors wish to thank Dr. G. E. Duvall for his helpful discussions.

\title{
Quantum-Mechanical Time Operator
}

\author{
M. RAZAVY \\ Department of Physics, University of Alberta, Edmonton, Alberta, Canada
}

(Received 6 February 1967; revision received 1 May 1967)

\begin{abstract}
A first integral of motion in classical dynamics which contains the time explicitly and is conjugate to the Hamiltonian is quantized. The resulting quantal operator is applied to study the time delay caused by scattering, and in a simple case the expectation value of this operator is obtained.
\end{abstract}

\section{INTRODUCTION}

$I^{N}$ $\mathrm{N}$ classical dynamics for a holonomic system with $n$ degrees of freedom, there are $2 n$ first integrals of motion. ${ }^{1}$ Among these are quantities like energy and angular momentum, which have been studied extensively in quantum mechanics. One of these first integrals which contains the time explicitly and is conjugate to the Hamiltonian is discussed in this paper. The quantal

\footnotetext{
* E. W. R. Steacie Fellow.

1 E. T. Whittaker, Analytical Dynamics (Cambridge University Press, Cambridge, England, 1937), p. 53.
}

form of this integral, which is called the time operator, is of interest in problems like the time delay in scattering, the lifetime of a metastable state, and the time-energy uncertainty relation. The present paper is concerned with the first problem, i.e., the time delay caused by scattering from a localized potential. The method developed here may be used to calculate time delay in scattering from short- or long-range potentials. While the construction of the quantal operator for localized Hamiltonians is straightforward, the resulting differential equation is quite complicated and generally of infinite order. 